\title{
BMJ Open Real-ear acoustical characteristics of impulse sound generated by golf drivers and the estimated risk to hearing: a cross-sectional study
}

\author{
Fei Zhao, ${ }^{1}$ Barry Bardsley ${ }^{2}$
}

To cite: Zhao F, Bardsley B. Real-ear acoustical characteristics of impulse sound generated by golf drivers and the estimated risk to hearing: a cross-sectional study. BMJ Open 2014;4: e003517. doi:10.1136/ bmjopen-2013-003517

- Prepublication history for this paper is available online. To view these files please visit the journal online (http://dx.doi.org/10.1136/ bmjopen-2013-003517).

Received 1 July 2013 Revised 12 November 2013 Accepted 13 November 2013

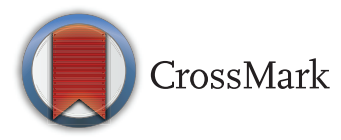

${ }^{1}$ Centre for Hearing and Balance Studies, University of Bristol, Bristol, UK ${ }^{2}$ College of Human and Health Science, University of Wales, Swansea, Swansea, UK

Correspondence to Dr Fei Zhao; Fei.Zhao@bristol.ac.uk

\section{ABSTRACT}

Objectives: This study investigated real-ear acoustical characteristics in terms of the sound pressure levels (SPLS) and frequency responses in situ generated from golf club drivers at impact with a golf ball. The risk of hearing loss caused by hitting a basket of golf balls using various drivers was then estimated.

Design: Cross-sectional study.

Setting: The three driver clubs were chosen on the basis of reflection of the commonality and modern technology of the clubs. The participants were asked to choose the clubs in a random order and hit six twopiece range golf balls with each club. The experiment was carried out at a golf driving range in South Wales, UK.

Participants: 19 male amateur golfers volunteered to take part in the study, with an age range of 19-54 years.

Outcome measures: The frequency responses and peak SPLs in situ of the transient sound generated from the club at impact were recorded bilaterally and simultaneously using the GN Otometric Freefit wireless real-ear measurement system. A swing speed radar system was also used to investigate the relationship between noise level and swing speed.

Results: Different clubs generated significantly different real-ear acoustical characteristics in terms of SPL and frequency responses. However, they did not differ significantly between the ears. No significant correlation was found between the swing speed and noise intensity. On the basis of the SPLs measured in the present study, the percentage of daily noise exposure for hitting a basket of golf balls using the drivers described above was less than $2 \%$.

Conclusions: The immediate danger of noise-induced hearing loss for amateur golfers is quite unlikely. However, it may be dangerous to hearing if the noise level generated by the golf clubs exceeded $116 \mathrm{dBA}$.

\section{INTRODUCTION}

The popularity of golf has seen significant increases in the past 25 years, particularly in Europe and the USA. In the UK alone figures from 2011 showed 1.3 million golf players and close to 3000 golf courses. ${ }^{1}$ By

\section{Strengths and limitations of this study}

- An innovative approach taken by using in-the-ear recordings that provide sound pressure levels at the tympanic membrane. This provides a more accurate estimation because it takes ear canal resonance properties into consideration.

- On the basis of the sound pressure levels measured in the present study, the risk of noise induced hearing loss for amateur golfers has been estimated and proven to be minimal.

- Only three golf clubs were examined, and a relatively small sample of participants was recruited in the study.

the nature of the game, golf requires the player to swing various types of clubs to hit balls into a relatively wide open space with the ultimate aim of sending the ball into a hole with the fewest number of shots. Therefore, golf is perceived as a low-risk sport compared with other highly competitive sports such as rugby, football, basketball or skiing that have higher injury rates.

However, several studies have shown insight into injuries related to golf. These range from common spine and upper or lower limb injuries to the less frequent injuries related to golf ball trauma. ${ }^{2}{ }^{3}$ In addition, golf has not been attributed to leisure noise exposure and noiseinduced hearing loss (NIHL) until recently. ${ }^{4}$ Noise can be described as sound at an intensity that can interfere with verbal communication and may cause discomfort of the ears or reduction of hearing sensitivity, defined as hearing damage. ${ }^{5} 6$ Any exposure to noise of significant intensity and duration increases the risk of ear damage and causes permanent hearing damage, known as NIHL. Both industrial noise exposure and noise in leisure are the major causes of avoidable permanent hearing loss throughout the world. Within the categories of leisure noise, noise exposure from 
sporting activities is a major recreational noise source relevant to various social activities, such as, motor sport, shooting and spectators at a football match. ${ }^{7-9}$

A study that investigated the potential hazards of modern driver golf clubs in damaging users' hearing from excessive exposure to loud sounds has highlighted this link. ${ }^{4}$ The authors reported on a case of a 55-year-old man who had appeared to have developed unilateral NIHL due to the exposure of loud noises generated from his driver golf club. When using a professional golfer, they found that many of the clubs generated sounds in excess of $120 \mathrm{dBA}$, particularly the thin-faced titanium drivers. Therefore, the authors recommended caution should be exercised by any golfers using the thin-faced titanium drivers to avoid damage to their hearing.

This raises a number of interesting questions. The use of a sound level meter (SLM) equidistant from the golfer provided information in the free field. However, in using an SLM it is unclear what acoustical effects the ear canal resonance would have on the noise generated. Further, the methods used previously did not provide ear specific information and whether the head shadow effect was implicated. In having one professional take part in the study it was unclear if this could be applied to amateur golfers, and whether the sound generated correlated with swing speed. So, there is uncertainty of the immediate and long-term dangers of such issues in golf, and a significant knowledge gap remains regarding effective guidance on hearing health awareness and prevention of this sport leisure noise damage to hearing. Therefore, the aim of this study was to further investigate the acoustical characteristics in situ using real-ear measurement system (REM) using various driver clubs. The relationship between swing speed and the noise levels generated was also investigated.

\section{MATERIAL AND METHOD \\ Participants}

Nineteen male amateur golfers volunteered to take part in the study, with an age range of 19-54 years (mean 38 years). Of them, 2 were left handed and 17 right handed. Ten had less than 10 years experience (53\%), 6 participants had golfing experience between 10 and 20 years $(31 \%)$, and 3 participants had more than 20 years experience $(16 \%)$. Approximately, $80 \%$ of participants reported that they play golf 1-2 times per week on separate days, and all but one participant used the driving range less than 3 times a week.

\section{Golf driver clubs}

The three driver clubs were chosen on the basis of reflection of the commonality and modern technology of the clubs together with consideration of their potentially high loudness levels as listed in the study by Buchanan et $a l^{4}$ Owing to the potential commercial dispute and conflicts of interest, the names of the manufacturers of the clubs were not disclosed, and consequently these differently branded clubs were coded as Club 1, Club 2 and Club 3 in the present study. However, this information can be discussed by contacting the authors if there is any concern about the potential hazard of hearing damage to the golf players. In addition, in accordance with experimental protocol, each participant was invited to bring their own driver and use it along with three other driver clubs.

\section{Measurement of real-ear responses}

Because of natural amplification of the external ear canal, for the purpose of this study, the real-ear acoustical characteristics in terms of sound frequency spectrum (ie, frequency response) and SPL were investigated using a probe microphone at a position near the eardrum. Following a warm-up period, the participants were asked to choose the clubs in a random order and hit six two-piece range golf balls with each club. The frequency responses and peak SPLs in situ of the transient sound generated from the club at impact were recorded bilaterally and simultaneously using the GN Otometric Freefit wireless REM. Probe placement was $25 \mathrm{~mm}$ along the external auditory canal. ${ }^{10}$ Room and probe tube calibration were carried out before performing the experiment for each participant.

All recordings were completed at a golf driving range in South Wales, UK.

For the purpose of measuring the real-ear acoustic characteristics in situ when the golfer was striking the ball, and to allow comparisons based on dexterity, a label of far and near-ear was used. This was implemented because it was unclear if there were differences between the right and left ears due to their distances from the ball, which would be determined by the position of each ear exposed to the ball depending on the golfer's dexterity. In essence the ball was positioned opposite the leading foot when using a driver club to promote an upward impact and trajectory. Therefore, right-handed golfers had their left ear defined as the near-ear, while the right ear was defined as the far-ear and vice versa for the left-handed golfers (figure 1).

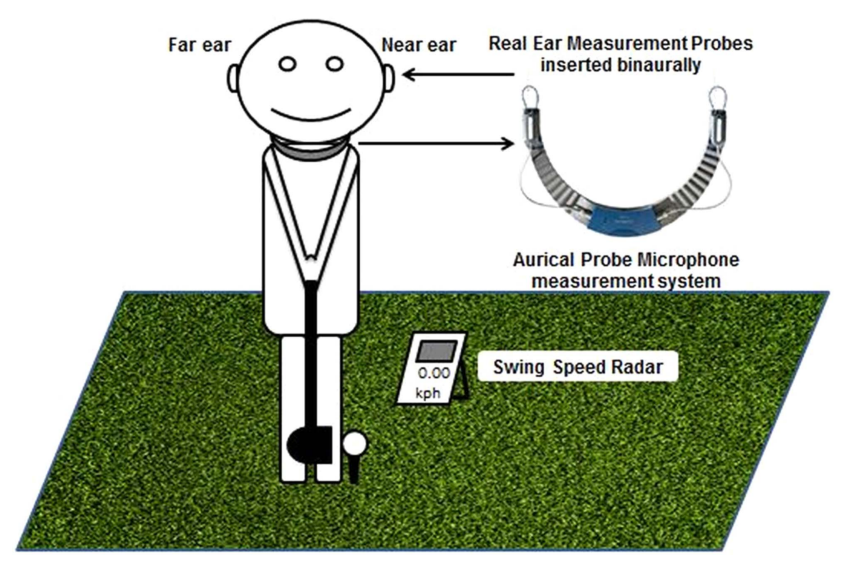

Figure 1 Diagram of experimental settings and equipment used. 
All recorded real-ear responses were reviewed and analysed. In the present study, the peak SPL was determined as the highest point of the curve, whereas the frequency response was referred to as the point corresponding to the measured peak SPL. Both peak SPL and frequency response were chosen from visual inspection by the authors, and then measured directly in the real-ear response curves.

\section{Swing speed measurement}

The participant swing speed was recorded using the Swing Speed Radar system. The unit houses a small microwave Doppler radar velocity sensor that provides an accurate measurement of club head speed at impact. The unit was positioned within 6 inches of the ball and in line with the swing plane.

\section{Ethics}

All participants were informed about the purpose, potential risks of the study, their roles and rights as participants, the nature of the study and issues of confidentiality and anonymity of the data. Participation was on a voluntary basis and information was provided regarding withdrawal from the study at any time. With the possibility of detecting hearing loss through the audiological evaluation, participants were provided with a copy of the hearing test and informed if they needed to consult with their general practitioner for further investigation.

\section{Statistical design and analyses}

Collected data was stored in an Excel database and all relevant analyses were carried out on Statistical Package for Social Science (SPSS) V.19.0 for Windows software. A one-way repeated measure analysis of variance (ANOVA) was performed to examine the effects of the golf clubs (ie, clubs 1, 2 and 3) and ear side (far-ear and near-ear) on the real-ear acoustical characteristics. The mean value and SD of the frequency responses and peak SPL were calculated and compared using a post hoc test (ie, Tukey HSD (Honestly Significant Difference)). A Pearson correlation analysis between swing speed and the real-ear acoustical characteristics was also performed. A $\mathrm{p}$ value $<0.05$ was considered to be statistically significant.

\section{RESULTS}

\section{Real-ear acoustical characteristics of sound impulses} generated by golf drivers

Figure 2 shows a scatter plot of the averaged SPLs obtained in individuals in the far-ears and near-ears generated by three golf drivers, which were used for the experiment. There was a significant correlation in the SPLs recorded between the far-ears and near-ears $(\mathrm{r}=0.77, \mathrm{p}<0.0005)$. The averaged SPLs were approximately 82-88 dBA while using clubs 2 and 3 (Club 2 far-ear and near-ear: 85.7 and $82.8 \mathrm{dBA}$; Club 3 far-ear

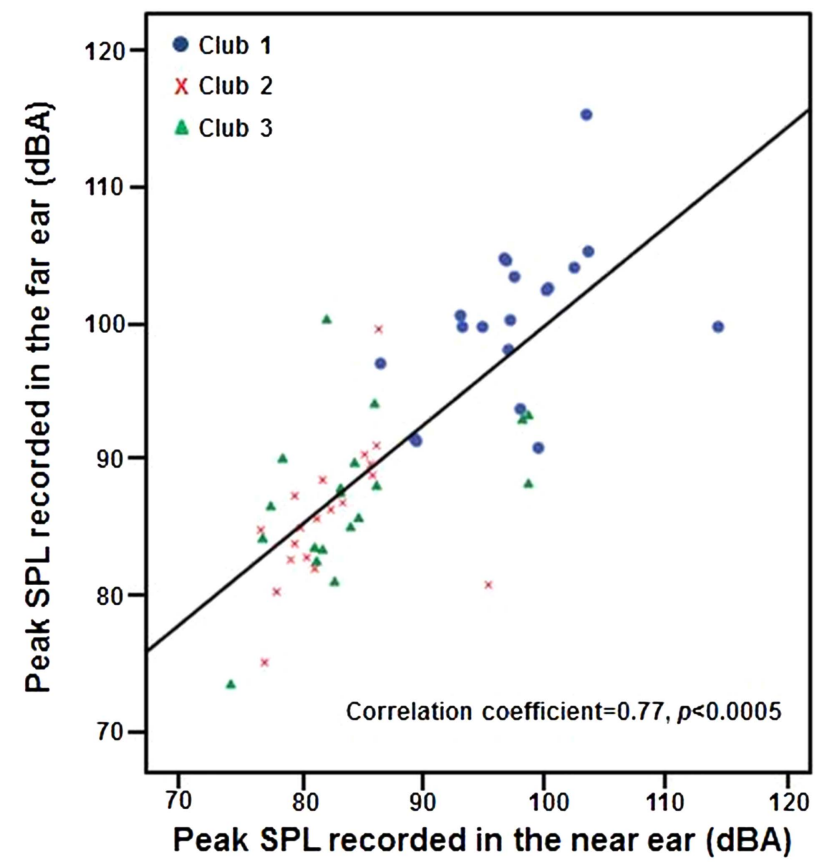

Figure 2 Scatter plot of averaged sound pressure levels (SPLs) obtained in individuals in the far-ears and near-ears generated by three golf drivers.

and near-ear: 87.0 and $84.8 \mathrm{dBA}$ ), whereas the averaged SPLs for Club 1 were 100 and 98.2 dBA on far-ears and near-ears, respectively (table 1 ). The repeated measures ANOVA was conducted to compare the sound levels generated from the different golf clubs and to investigate the $\mathrm{dB}$ recorded in each ear. The repeated measures ANOVA results showed that there were significant differences in the SPLs obtained from clubs 1, 2 and 3 (Wilks' $\lambda=0.06$, F $(2,17)=134.33$; $\mathrm{p}<0.0005)$. However, no significant differences were found in the SPLs between the far-ears and the near-ears $(\mathrm{F}(1,18)=3.48, \mathrm{p}=0.08)$. Further analysis showed that the SPLs generated by Club 1 were significantly greater than those found in clubs 2 and $3(\mathrm{p}<0.0005)$. In contrast, there was no significant difference in the SPLs between Club 2 and Club 3.

Furthermore, frequency response analysis showed that the different golf drivers had different frequency

\begin{tabular}{|c|c|c|c|}
\hline & Club 1 & Club 2 & Club 3 \\
\hline \multicolumn{4}{|c|}{ Sound pressure level in the ear canal (dBA) } \\
\hline Far-ear & $100.3 \pm 1.3$ & $85.7 \pm 1.2$ & $87.0 \pm 1.3$ \\
\hline Near-ear & $98.2 \pm 1.4$ & $82.8 \pm 1.0$ & $84.8 \pm 1.6$ \\
\hline \multicolumn{4}{|c|}{ Frequency response in the ear canal $(\mathrm{kHz})$} \\
\hline Far-ear & $2.49 \pm 0.03$ & $3.60 \pm 0.01$ & $3.60 \pm 0.09$ \\
\hline Near-ear & $2.53 \pm 0.03$ & $3.37 \pm 0.11$ & $3.44 \pm 0.08$ \\
\hline $\begin{array}{l}\text { Swing speed } \\
\text { (kph) }\end{array}$ & $166.5 \pm 15.4$ & $169.0 \pm 15.4$ & $168.9 \pm 17.3$ \\
\hline
\end{tabular}




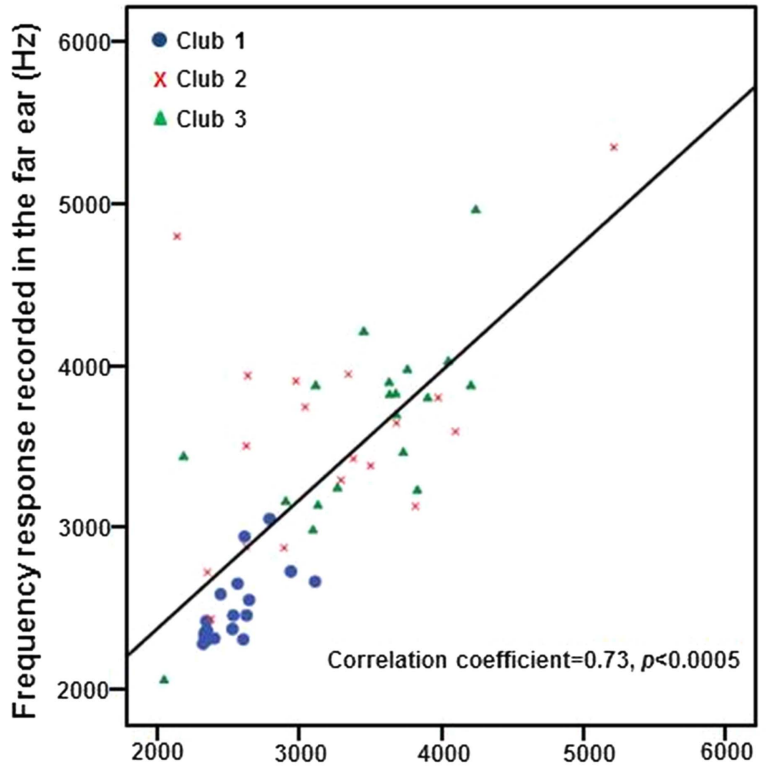

Frequency response recorded in the near ear $(\mathrm{Hz})$

Figure 3 Scatter plot of averaged frequency responses obtained in individuals in the far-ears and near-ears generated by three golf drivers.

characteristics. As shown in figure 3, there was a significant correlation in the peak frequencies recorded between the far-ears and near-ears $(\mathrm{r}=0.73, \mathrm{p}<0.0005)$. Clubs 2 and 3 had similar peak frequency characteristics around $3.5 \mathrm{kHz}$ when striking the balls, while the average frequency response characteristic of Club 1 was $2.5 \mathrm{kHz}$ on either the far-ear or the near-ear. Although no significant differences were found in frequency responses between the far-ears and the near-ears ( $\mathrm{F}(1$, $18)=2.18, p=0.16$ ), the repeated measures ANOVA results showed that the peak frequencies differed significantly among the three clubs ( $\mathrm{F}(2,17)=38.72, \mathrm{p}<0.0005)$. Further analysis showed that the frequency responses found in the Club 1 were significantly lower than those in Clubs 2 and $3(\mathrm{p}<0.0005)$. In contrast, there was no significant difference in the frequency responses between Club 2 and Club 3.

\section{Correlation between sound intensity and swing speed}

Table 1 shows the average swing speed when using different golf drivers. A one-way ANOVA test showed that there were no significant differences in swing speeds among clubs 1, 2 and 3 (Club 1 vs Club 2 vs Club 3: 166.5 vs 169.0 vs $168.9, \mathrm{~F}=0.18 ; \mathrm{p}=0.84)$. The relationship between sound intensity ( $\mathrm{dB}$ level) and swing speed $(\mathrm{kph})$ was investigated using the Pearson productmoment correlation coefficient. There was a small, positive correlation between the two variables, but no statistical significance was found $\quad\left(\mathrm{r}_{\mathrm{far}-\mathrm{ear}}=0.13, \mathrm{p}=0.32\right.$; $\left.\mathrm{r}_{\text {near-ear }}=0.05, \mathrm{p}=0.72\right)$.

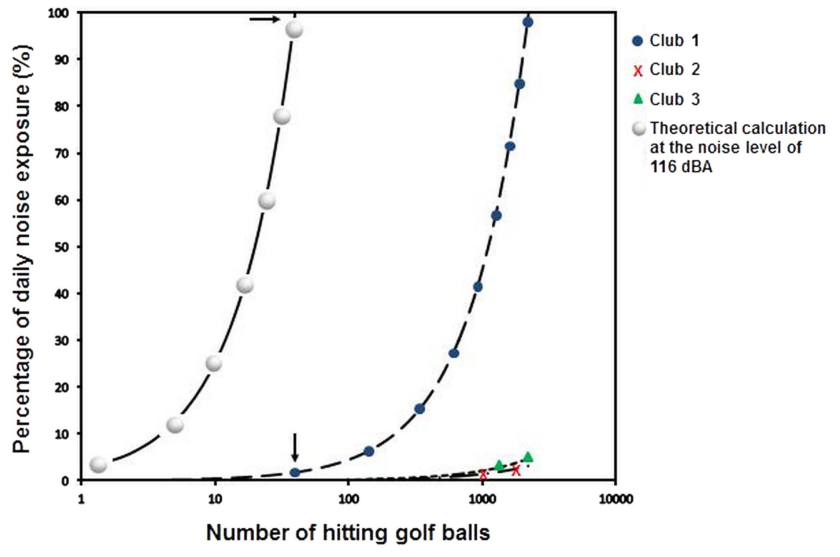

Figure 4 Percentage of daily noise exposure when hitting golf balls using the drivers. Arrow label $(\rightarrow)$ : the percentage of daily noise exposure per basket (40 golf balls).

Hearing risk estimation by calculating the percentage of daily noise exposure for hitting a basket of golf balls

Hearing risk was estimated by calculating the percentage of daily noise exposure for hitting a basket of golf balls using those drivers described above. The sound duration of striking a golf ball was measured by recording the sound waveforms from the beginning of the impulse sound until it fell away when crossing the baseline. On average, the sound duration of striking a golf ball was recorded as approximately $0.5 \mathrm{~s}$. If hitting a basket of golf balls, which are typically 40 in number, the total sound exposure duration was $20 \mathrm{~s}$ (ie, $0.00556 \mathrm{~h}$ ).

In the present study, in order to estimate hearing risk, the damage criteria of $85 \mathrm{dBA}$ for a maximum $8 \mathrm{~h}$ period advocated by the National Institute for Occupational Safety and Health $(\mathrm{NIOSH})^{11}$ was adapted with an exchange rate of $3 \mathrm{~dB}$ and using the A-weighting scale. Thus, the following formula was used

$$
\begin{aligned}
& \text { Daily noise dose } \% \\
& =\mathrm{T}_{1}(\text { total exposure duration }) \\
& / \mathrm{T}(\text { reference duration }) \times 100
\end{aligned}
$$

where

$$
\left.\mathrm{T}=8 / 2^{\wedge}(\mathrm{SPL}-85) / 3\right) \text { and } \mathrm{T} 1=0.00556 .
$$

Because the sound intensities generated by the golf clubs were recorded and measured in the ear canal, in order to compare them with the NIOSH standard, the transformation from real-ear SPLs to free-field equivalent values was performed according to ISO $11904-1^{12}$ (ie, inversion of the average field-to-eardrum transfer function).

On the basis of the SPLs measured in the present study, the percentage of daily noise exposure for hitting a basket of golf balls using the drivers described above were minimal $(0.05-1.8 \%$ figure 4$)$. For instance, when using Club 1, the noise of hitting approximately 2200 balls with SPLs around $99 \mathrm{dBA}$ would reach $100 \%$ of the 
daily noise exposure dose. In addition, by theoretical calculation, the noise level of hitting a basket of balls (40) at $116 \mathrm{dBA}$ appeared to reach the $100 \%$ exposure limit.

\section{DISCUSSION}

Although a small sample of clubs was used in the present study, and therefore the findings cannot be attributed to all driver clubs on the market, it does demonstrate that there is significant variance in the output generated from thin-faced titanium drivers used in the present study, showing significant differences in the intensity and frequency characteristics obtained from this type of club compared with the others. It implies that golf driver clubs differ in terms of a potential risk to hearing damage. On the basis of the results derived from the present study with 19 amateur golfers, the noise levels generated on the whole (approximately $90 \mathrm{~dB}$ on average) do not indicate immediate risk. This is consistent with previous studies, ${ }^{13} 14$ which show $80-94 \mathrm{~dB}$ when measuring accurate hitting sounds using the ArtemiS system, which includes a binaural headset in connection with integrated microphones near the opening of the ear canal. However, the current result is contrasted with an earlier report using a professional golfer to hit three balls with six thin-faced titanium drivers, ${ }^{4}$ which demonstrated a significantly highnoise level in the region of 120-130 dBA, although only a few balls were hit at these intensities in the present study.

Such a discrepancy is mainly due to different measurement methods employed between the study carried out by Buchanan et al and the present study. REM used in the present study is a common method for measuring SPL near the tympanic membrane. ${ }^{15}$ It offers an accurate and objective approach including the individual's real-ear acoustic characteristics. By contrast, in the study by Buchanan $e t a l,{ }^{4}$ the SPL was measured using an SLM in the free-field equidistant from the golfer. The limitation of this measurement is not accurate because the acoustical effects of the ear canal resonance are not taken into account. Moreover, the other key issue is that the SPLs measured in the free field are not the actual SPLs in the ear canal because the baffle effect of the head and torso is also important for measuring sound transfer from the free field to the ear. In addition, the skills and power possessed by professional golfers may play a role in generating louder SPLs than is the case for amateur golfers.

Although various studies have suggested that exposure to stimuli exceeding the $100 \%$ daily noise dose would cause hearing damage, including a hearing sensitivity reduction, tinnitus, hyperacusis and distortion, ${ }^{5} 616$ recent research argued the appropriateness of applying industrial risk criteria with recreational noise exposure, because these standards were developed specifically for spectrally dense industrial noise with limited dynamic range as an exposure dose for an $8 \mathrm{~h}$ workday. Therefore, comparisons with industrial standards have to be made with caution. Nevertheless, according to the intensity and noise exposure dose calculation found in the present study, the risks are, on the whole, negligible, that is, the golf players, particularly amateur players are most unlikely to have hearing damage. However, it is noteworthy that some golf clubs can generate extremely high levels of noise, such as at the maximum value of 123 dBA recorded from Club 1 used in the study. With such high-noise intensity exposure, it is most likely to pose a significant risk of NIHL if the driver is used frequently. This would fit with the case study of an amateur golfer presenting with significant hearing loss. ${ }^{4}$

Furthermore, the sound of a golf club hitting a golf ball is one of the influencing factors at a subconscious level on a golfer's perception of quality and his/her choice of equipment, because it provides information on whether the ball was hit correctly. Therefore, golf club manufactures tend to make new models of golf driver clubs using various materials (such as titanium) not only to achieve longer distances, but also to create unique sound characteristics to attract attention. The study by Roberts et $a l^{17}$ investigated the relationship between the impact sound and elite golfers' subjective perceptions of a shot. They found a strong relationship between the characteristics of sharpness and loudness of sound, and pleasantness and liveliness of perception. Significantly positive correlations were also obtained between the subjective ratings and parameters of the impact sound such as SPL, loudness level and sharpness. This suggests that the golfers' perceptions are influenced by frequency components, loudness and duration.

In addition, the fast Fourier transform analysis in the previous study ${ }^{13}$ showed different sounds in terms of frequency characteristics and reverberation of the tonal components generated by different clubs when they hit the balls. This is in keeping with the findings of the present study, demonstrating that the peak frequency characteristics recorded from golf driver clubs varied significantly between 2.5 and $3.5 \mathrm{kHz}$. This is associated with the design (eg, the structure of the club head) and materials used to make these modern clubs, which have been created by the manufactures in order to achieve a more balanced frequency distribution and thus a better sound quality. However, with such clubs, they are likely to create louder hitting sounds than conventional golf clubs, which potentially causes damage to golfers' hearing.

In the present study, the results did not show evidence of a head shadow effect insofar as there was no significant difference in sound intensity between the recordings from the near-ears and far-ears. There is some controversy about asymmetrical noise exposure even though both ears are exposed directly to impact sound sources. $(8,18,19$ and 20) Some previous studies argued that left ear NIHL are predominantly common in the army, because the ear opposite to the dominant hand sustains over exposure mainly due to the shooting posture. ${ }^{18}{ }^{19}$ However, a recent study on hearing performance after recreational firearm use did not reveal a significant preference in terms of temporary threshold shift between ears, irrespective of the 
dominant hand. ${ }^{8}$ The other study by Job $e t a l^{20}$ showed that the asymmetry of hearing thresholds between the left ear and right ear was not associated with the participant's shooting posture. They suggested that it is most likely due to different intrinsic characteristics in each side of the ears. In addition, no head diffraction effect found in this study can be explained as rapid movement of the torso and the head when swinging the golf club, which means that exposure to the impact noise generated is spread across the two ears. When the torso is uncoiling, the body and head move to a position where both ears face directly into the impact sound sources. Therefore, one side of the ear is overly exposed, and as a consequence, a unilateral hearing loss is unlikely to be developed from playing golf.

Swing speed did not show any significant correlations with either acoustical characteristics measured in the golf driver clubs (ie, intensity levels and peak frequencies). Golfers' skill and power (particularly among professional golfers) may be one of the important influencing factors on swing speed. It is noteworthy that another important factor is the area of the club face hitting the ball to create an impact because the face of the driver club has a 'sweet spot' where the trampoline effect is optimal. The trampoline effect refers to a pronounced deformation of the club face upon impact followed by a quick restoration to its original dimensions, acting like a slingshot. When the club face hits the ball right on the sweet spot, it results in very high ball speeds. In the present study, it is impossible for the participants to control this factor in order to deliver the same performance with each strike. An investigation of strikes out of the heel, toe and sweet spot using an automated and controlled swing motion with a robot $^{21}$ would add valuable insight into this enquiry, particularly when comparisons are made between amateur and professional golfers.

\section{CONCLUSION}

Different clubs generated significantly different real-ear acoustical characteristics in terms of SPLs and frequency responses. On the basis of the SPLs measured in the present study, the immediate danger of NIHL for amateur golfers is quite unlikely. However, it is most likely to pose a significant risk of NIHL if the driver generates high-noise intensity greater than $116 \mathrm{dBA}$. The provision of detailed information on a club's acoustical characteristics may help consumers choose the appropriate device for their needs, particularly for people who are prone to hearing damage. A longitudinal study monitoring hearing thresholds would provide a valuable insight in terms of the long-term risks to hearing.

Acknowledgements The authors would like to thank two reviewers and managing editor for their helpful suggestions. The authors would also like to acknowledge GN Otometrics UK for providing the Freefit wireless Real Ear Measurement System and technical support during the study. Mrs Norma Meechem kindly provided help with proof reading.

Contributors BB developed the idea, designed the study, collected the data, analysed the data and wrote the article. FZ wrote the grant application, designed the study, collected the data and revised the artilce. FZ is the guarantor.

Funding This study was supported by the Great Britain Sasakawa Foundation (B68).

\section{Competing interests None.}

Ethics approval Graduate School of Education (GSoE), University of Bristol.

Provenance and peer review Not commissioned; externally peer reviewed.

Data sharing statement Additional data are available by emailing Fei. Zhao@bristol.ac.uk. These data are additional information from a questionnaire regarding golf.

Open Access This is an Open Access article distributed in accordance with the Creative Commons Attribution Non Commercial (CC BY-NC 3.0) license, which permits others to distribute, remix, adapt, build upon this work noncommercially, and license their derivative works on different terms, provided the original work is properly cited and the use is non-commercial. See: http:// creativecommons.org/licenses/by-nc/3.0/

\section{REFERENCES}

1. KMPG. Golf participation in Europe 2011. http://www.kpmg.com/Global/ en/industry/real-estate/Documents/golf-participation-in-europe.pdf

2. McHardy A, Pollard H, Luo KH. Golf injuries: a review of the literature. Sports Med 2006;36:171-87.

3. Parziale JR, Mallon WJ. Golf Injuries and rehabilitation. Phys Med Rehabil Clin N Am 2006;17:589-607.

4. Buchanan MA, Prinsley PR, Wilkinson JM, et al. Is golf bad for your hearing? BMJ 2008;337:1437-8.

5. Maltby M. Noise induced hearing loss. In: Maltby M. ed Occupational audiometry-monitoring and protecting hearing at work. Oxford: Elsevier Ltd, 2005:3-19.

6. Zhao F, Manchaiah V, French D, et al. Music exposure and hearing disorders: an overview. Int J Audiol 2010;49:54-64.

7. Bentley S. Noise induced deafness. Br J Sport Med 1978;12:160.

8. Bapat U, Tolley N. Temporary threshold shift due to recreational firearm use. J Laryngol Otol 2007;121:927-31.

9. Engard DJ, Sandfort DR, Gotshall RW, et al. Noise exposure, characterization, and comparison of three football stadiums. J Occup Environ Hyg 2010;7:616-21.

10. British Society of Audiology. Guidance on the use of real ear measurement to verify the fitting of digital signal processing hearing aids. 2007. http://www.thebsa.org.uk/docs/RecPro/REM.pdf

11. National Institute for Occupational Safety and Health (NIOSH). Criteria for a recommended standard: occupational noise exposure, revised criteria 1998. DHHS (NIOSH) Pub. No. 98-126. Washington, DC: US Department of Health and Human Services, 1998.

12. ISO 11904-1. Acoustics-determination of sound immission from sound sources placed close to the ear Part 1: technique using a microphone in a real ear (MIRE technique), Switzerland: ISO.

13. Application Note: analysis of sounds caused by a golf club hitting the ball. http://www.head-acoustics.de/downloads/eng/application notes/Golfclubs 06 11e.pdf

14. Ellis B. Which is king of clubs in the noise stakes? 2009. http://www. derby.ac.uk/news/which-is-king-of-clubs-in-the-noise-stakes

15. Hodgetts WE, Rieger JM, Szarko RA. The effects of listening environment and earphone style on preferred listening levels of normal hearing adults using an MP3 player. Ear Hear 2007;28:290-7.

16. Worthington DA, Siegel JH, Wilber LA, et al. Comparing two methods to measure preferred listening levels of personal listening devices. J Acoust Soc Am 2009;125:3735-41.

17. Roberts JR, Jones R, Mansfield NJ, et al. Evaluation of impact sound on the 'feel' of a golf shot. $J$ Sound Vibration 2005;287:651-66.

18. Prosser S, Tartari MC, Arslan E. Hearing loss in sports hunters exposed to occupational noise. Br J Audiol 1988;22:85-91.

19. Pelausa EO, Abel SM, Simard J, et al. Prevention of noise-induced hearing loss in the Canadian military. J Otolaryngol 1995;24:271-80.

20. Job A, Grateau P, Picard J, et al. Intrinsic differences in hearing performances between ears revealed by the asymmetrical shooting posture in the army. Hear Res 1998;122:119-24.

21. Ming A, Henmi M, Xu C, et al. A new motion controlled method for golf swing robot hitting ball. IEEE/ASME International Conference on Advanced Intelligent Mechatronics Proceedings 2005;1517-22. 